\title{
The beat Cepheids in the Magellanic Clouds: an analysis from the EROS-2 database da $^{\star \star}$
}

J. B. Marquette ${ }^{1}$, J. P. Beaulieu ${ }^{1}$, J. R. Buchler ${ }^{2}$, R. Szabó ${ }^{3}$, P. Tisserand ${ }^{4,5}$, S. Belghith ${ }^{1}$, P. Fouqué ${ }^{6}$, É. Lesquoy ${ }^{1,5}$, A. Milsztajn ${ }^{5, \star \star \star}$, A. Schwarzenberg-Czerny ${ }^{7,8}$, C. Afonso ${ }^{3,9}$, J. N. Albert ${ }^{10}$, J. Andersen ${ }^{11}$, R. Ansari ${ }^{10}$, É. Aubourg ${ }^{5}$, P. Bareyre ${ }^{5}$, X. Charlot ${ }^{5}$, C. Coutures ${ }^{1,5}$, R. Ferlet ${ }^{1}$, J. F. Glicenstein ${ }^{5}$, B. Goldman ${ }^{5,9}$, A. Gould ${ }^{12}$, D. Graff ${ }^{12,13}$, M. Gros ${ }^{5}$, J. Haïssinski ${ }^{10}$, C. Hamadache ${ }^{5}$, J. de Kat ${ }^{5}$, L. Le Guillou ${ }^{5,14}$, C. Loup ${ }^{1,15}$, C. Magneville ${ }^{5}$, É. Maurice ${ }^{16}$, A. Maury ${ }^{17,18}$, M. Moniez ${ }^{10}$, N. Palanque-Delabrouille ${ }^{5}$, O. Perdereau ${ }^{10}$, Y. R. Rahal ${ }^{10}$, J. Rich ${ }^{5}$, M. Spiro ${ }^{5}$, and A. Vidal-Madjar ${ }^{1}$

(Affiliations can be found after the references)

Received 21 August 2008 / Accepted 13 November 2008

ABSTRACT

Context. A number of microlensing dark-matter surveys have produced tens of millions of light curves of individual background stars. These data provide an unprecedented opportunity for systematic studies of whole classes of variable stars and their host galaxies.

Aims. We aim to use the EROS-2 survey of the Magellanic Clouds to detect and study the population of beat Cepheids (BCs) in both Clouds. BCs pulsating simultaneously in the first overtone and fundamental modes (FO/F) or in the second and first overtone modes (SO/FO) are of particular interest.

Methods. Using special software designed to search for periodic variables, we have scanned the EROS-2 data base for variables in the typical period range of Cepheids. Metallicities of $\mathrm{FO} / \mathrm{F}$ objects were then calculated from linear nonadiabatic convective stellar models.

Results. We identify $74 \mathrm{FO} / \mathrm{F} \mathrm{BCs}$ in the LMC and 41 in the SMC, and 173 and 129 SO/FO pulsators in the LMC and SMC, respectively; 185 of these stars are new discoveries. For nearly all the FO/F objects we determine minimum, mean, and maximum values of the metallicity.

Conclusions. The EROS data have expanded the samples of known BCs in the LMC by $31 \%$, in the SMC by $110 \%$. The FO/F objects provide independent measures of metallicities in these galaxies. The mean value of metallicity is 0.0045 in the LMC and 0.0018 in the SMC.

Key words. Magellanic Clouds - Cepheids

\section{Introduction}

Over the last decade, large microlensing surveys have been conducted to search for planetary- to stellar-mass baryonic dark matter in the Milky Way. While, interestingly, the original search was in fact negative (Tisserand et al. 2007), the resulting tens of millions of homogeneous, well-sampled light curves of the background stars have stimulated many new programmes on variable stars. In particular, specific stellar populations like Cepheids can now be studied far more thoroughly, particularly in the Magellanic Clouds. Among these objects, the subsample of double-mode or beat Cepheids (hereafter BCs) is of particular importance in gaining information on the structure and evolution of massive stars.

According to the General Catalogue of Variable Stars (Samus et al. 2004), the Galactic sample of known BCs is rather small: up to now, only 16 objects of this type have been identified. In the Large (LMC) and Small (SMC) Magellanic Clouds the situation is more favourable, thanks in particular to the work

* Based on observations made by the EROS-2 collaboration with the MARLY telescope at the European Southern Observatory, La Silla, Chile.

* Data tables are only available in electronic form at the CDS via anonymous ftp to cdsarc.u-strasbg.fr (130.79.128.5) or via

http: //cdsweb.u-strasbg.fr/cgi-bin/qcat?J/A+A/495/249

$\star \star \star$ Deceased. of the EROS-1 (Beaulieu et al. 1995, 1997), MACHO (Alcock et al. 1995, 1999), and OGLE (Udalski et al. 1999; Soszynski et al. 2000) groups. EROS-1 detected one BC in the LMC and 11 in the SMC, while the MACHO group found $45 \mathrm{BCs}$ in the LMC. The OGLE collaboration reported the discovery of 93 BCs in the SMC and 76 in the LMC, very recently extended by the OGLEIII data to a region of $40 \mathrm{deg}^{2}$ of the LMC (Soszynski et al. 2008). This thorough analysis yielded 266 BCs of different types discussed below. The present study will report results on an even larger region of $88 \mathrm{deg}^{2}$ in the LMC and $10 \mathrm{deg}^{2}$ in the SMC.

A BC pulsates either in the first overtone and fundamental modes $(\mathrm{FO} / \mathrm{F})$, or in the second and first overtone modes $(\mathrm{SO} / \mathrm{FO})^{1}$. The studies listed above clearly established that the period ratio (higher to lower mode) of the $\mathrm{FO} / \mathrm{F}$ pulsators is around 0.72 , while that of $\mathrm{SO} / \mathrm{FO}$ objects is near 0.80 . For the $\mathrm{FO} / \mathrm{F}$ pulsators it is well known that the period ratio depends on the metallicity $Z$, as seen e.g. in the Petersen diagram (Petersen 1973 ) of the period ratio vs. the period of the lower mode. From OGLE photometry and stellar atmosphere models, Kovács (2008) showed that, in both Clouds, the average metallicity of FO Cepheids is lower than those pulsating in the fundamental mode.

The chemical enrichment history of the Magellanic Clouds has been recently reexamined by Carrera et al. (2008a,b), who

\footnotetext{
1 Two cases are known of pulsations in the first and third overtones (Soszynski et al. 2008).
} 
used the infrared CaII triplet as a metallicity indicator for red giant branch stars (Carrera et al. 2007). Leisy \& Dennefeld (2006) used planetary nebulae to trace the heavy elements in the Magellanic Clouds. They concluded that only Ar is a suitable tracer of the metallicity of the progenitor stars, instead of an average of several different elements. Idiart et al. (2007) also studied the chemical evolution of the SMC from planetary nebulae and concluded that the star formation histories of the LMC and SMC are different from each other and in many respects still controversial.

The situation is complicated by the fact that metallicity measurements in the Magellanic Clouds yield many different values, depending both on the location and age of the stars studied. Thus, a single firm value of the metallicity in these galaxies cannot be assigned. Abundances of $\alpha$-, iron-peak and $s$-process elements in individual stars are now being measured with highresolution spectrometers, for example by Pompéia et al. (2008), but it remains important to contribute to these efforts by adding a new and independent way to measure the metallicity, as we do below.

In a similar study, Beaulieu et al. (2006) deduced a metallicity gradient in M33, only from the pulsational properties of 5 newly discovered BCs in M33 and with the help of stellar pulsation theory and mass-luminosity relations derived from evolutionary tracks. The result was in good agreement with the standard spectroscopic metallicity gradients as determined from $\mathrm{H}$ II regions, early-B supergiant stars, and planetary nebulae. BCs are thus a new and independent probe of galactic metallicity. In this paper we present the results of a systematic search for and analysis of BCs in the Magellanic Clouds from the EROS-2 database. Numerous previously unknown objects are detected, and metallicities are derived from the properties of FO/F BCs in both Clouds.

\section{Observational setup and $B C$ search}

The EROS-2 experiment was conducted between July 1996 and March 2003 using the dedicated MARLY telescope $(1 \mathrm{~m}$ Ritchey-Chrétien, f/5.14) at ESO, La Silla. A dichroic beamsplitter allowed simultaneous imaging in two broad nonstandard passbands, $B_{\mathrm{E}}$ (4200-7200 ̊, "blue" channel) and $R_{\mathrm{E}}$ (6200-9200 ^̊, "red" channel). Each camera contained a mosaic of eight $2 \mathrm{~K} \times 2 \mathrm{~K}$ LORAL CCDs with a pixel size of $0.6^{\prime \prime}$ and a field of view of $0.7^{\circ}$ (right ascension) $\times 1.4^{\circ}$ (declination).

The light curves of individual stars were constructed from fixed positions on templates using special-purpose software for photometry of EROS-2 images called PEIDA (Ansari 1996). This resulted in a database of tens of millions of light curves, which will be publicly available in the future from the CDS through a dedicated server. For a typical object, 800 to 1000 epochs of observation are available.

Each light curve is named according to the rules given by Derue et al. (2002). As an example, the star J053929-703819 with J2000 equatorial coordinates of 05:39:29.15, -70:38:19.2 is also referred to by the internal EROS-2 number $1 \mathrm{~m} 059115619$ (the $5619^{\text {th }}$ star in the field $\operatorname{lm} 059$, CCD 1, quarter 1). In the following, we refer to the objects by their internal number.

A systematic search for periodic objects using the $B_{\mathrm{E}}$ observations was performed among stars detected as potentially variable by an automatic time series analysis pipeline, based on software developed by Beaulieu et al. (1997) and Schwarzenberg-Czerny (2003), and on an analysis of variance $(\mathrm{AoV})$ method. For each periodic pulsator we constructed
Table 1. Contents of the data tables for the new BCs in the LMC and SMC.

\begin{tabular}{|c|c|}
\hline Column name & Comments \\
\hline EROS-2 ID & Object identifier $^{a}$ \\
\hline RA & $\mathrm{J} 2000$ right ascension \\
\hline DEC & J2000 declination \\
\hline$P_{0}$ & Period (days) of the $1^{\text {st }}$ mode of pulsation \\
\hline$P_{1}$ & Period (days) of the $2^{\text {nd }}$ mode of pulsation \\
\hline$P_{10}$ & Period ratio $P_{1} / P_{0}$ \\
\hline$Z_{\max }$ & Maximum metallicity $^{b}$ \\
\hline$Z_{\min }$ & Minimum metallicity $^{b}$ \\
\hline$Z_{\text {mean }}$ & Mean metallicity ${ }^{b}$ \\
\hline$R_{0}$ & Fourier coefficient in $B_{\mathrm{E}}$ \\
\hline$R_{1}^{1}$ & idem $^{c}$ \\
\hline$\Phi_{1}^{1}$ & idem $^{c}$ \\
\hline$R_{21}^{1}$ & idem $^{c}$ \\
\hline$\Phi_{21}^{1}$ & idem $^{c}$ \\
\hline$R_{31}^{1}$ & idem $^{c}$ \\
\hline$\Phi_{31}^{1}$ & idem $^{c}$ \\
\hline$R_{1}^{2^{1}}$ & idem $^{c}$ \\
\hline$R_{21}^{2}$ & idem $^{c}$ \\
\hline$\Phi_{21}^{2}$ & idem $^{c}$ \\
\hline$R_{31}^{2}$ & idem $^{c}$ \\
\hline$\Phi_{31}^{2}$ & idem $^{c}$ \\
\hline
\end{tabular}

${ }^{a}$ As defined by Derue et al. (2002); ${ }^{b}$ for FO/F pulsators only; ${ }^{c}$ superscript $i$ refers to the $i^{\text {th }}$ pulsation mode.

a Fourier decomposition of the light curve, i.e. the signal was represented by $R(t)=R_{0}+\sum_{i=1}^{i=5} R_{i} \cos \left(\frac{2 \pi}{P} i\left(t-t_{0}\right)+\Phi_{i}\right)$ (Simon \& Lee 1981). The quantities $R_{\mathrm{k} 1}=R_{\mathrm{k}} / R_{1}$ and $\Phi_{\mathrm{k} 1}=\Phi_{\mathrm{k}}-k \Phi_{1}$ $(1<k \leq 3)$ were defined as usual.

The maximum of the statistic $\Theta_{\mathrm{AoV}}$ as defined by Schwarzenberg-Czerny (2003) was used as a quality parameter for the periodograms, which were stored when $\Theta_{\mathrm{AoV}} \gtrsim 20$. In this way, a total of about 80000 variables was found in both Clouds. The Fourier model was then subtracted from the observational data before a second step of period search by the same pipeline. Double-mode objects thus emerged quite clearly from the resulting periodograms. Potential BCs were identified by having period ratios near 0.73 and 0.80 for $\mathrm{FO} / \mathrm{F}$ and $\mathrm{SO} / \mathrm{FO}$ pulsators, respectively.

To construct the final samples, the light curves of all candidates (folded according to the first mode of pulsation) were inspected visually, using the versatile tool TOPCAT developed by the Starlink consortium ${ }^{2}$ and customized to display SuperMongo plots. On a typical BC light curve, folded according to either the first or second period, the beat phenomenon is easily recognised because the light curve is broader than that of a single mode Cepheid. This was our ultimate criterion of selection for objects with the lowest values of $\Theta_{\mathrm{AoV}}$.

Figure 1 shows six typical LMC objects, alternately of FO/F and $\mathrm{SO} / \mathrm{FO}$ type. The two first are firm detections with high values of $\Theta_{\mathrm{AoV}}$ for the second pulsation mode, the two in the middle are detected more marginally, and the two last have been rejected as having too low values of $\Theta_{\mathrm{AoV}}$. The left-hand panels show the folded light curves (blue magnitude vs. period of the second mode of pulsation, in days), after subtraction of the Fourier model of the first mode. The right-hand panels show the corresponding periodograms $\left(\Theta_{\mathrm{AoV}}\right.$ vs. frequency of the second mode period). In each of these periodograms the highest peak corresponds to the most probable frequency.

2 Available at http://www. star.bris.ac.uk/ mbt/topcat/ 

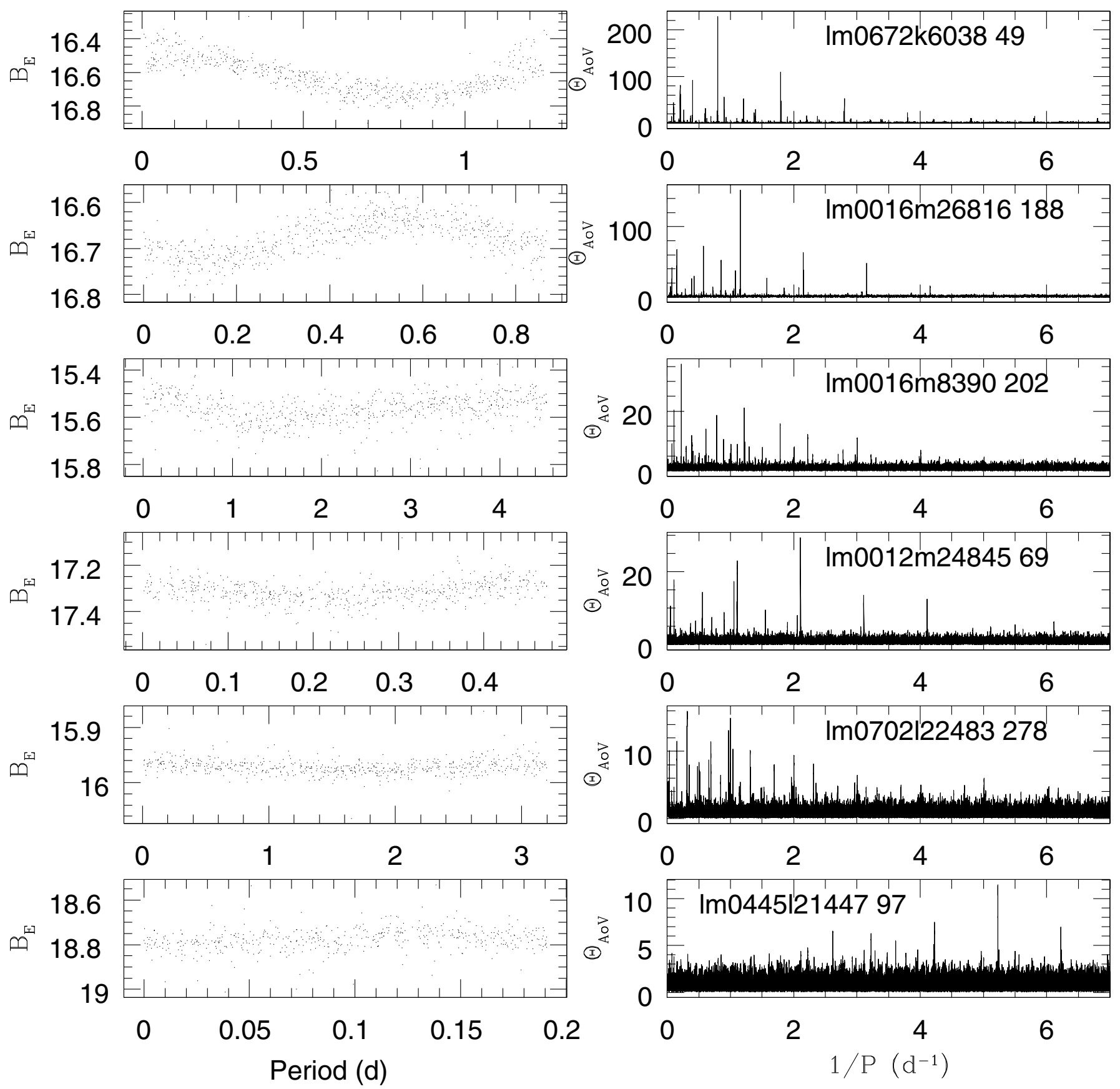

Fig. 1. Folded light curves and corresponding periodograms of 6 typical LMC BCs, alternately FO/F and SO/FO objects, from the most significant (top) to non-significant secondary modes (bottom). Left: $B_{\mathrm{E}}$ magnitude vs. period (days); right: $\Theta_{\mathrm{Aov}}$ for the second pulsation vs. frequency $\left(\right.$ days $^{-1}$ ). The name of each object is given in the right-hand panels, together with the maximum $\Theta_{\mathrm{Aov}}$ for the first pulsation mode. See text for details.

\section{General analysis and determination of the metallicities}

The visual inspection yielded $74 \mathrm{FO} / \mathrm{F}$ pulsators in the LMC and 41 in the SMC as well as $173 \mathrm{SO} / \mathrm{FO}$ stars in the LMC and 129 in the SMC. The pertinent data for these stars are given in separate tables for the $\mathrm{FO} / \mathrm{F}$ and $\mathrm{SO} / \mathrm{FO}$ pulsators in each galaxy (LMC and SMC) which are only available in electronic form at the CDS. Table 1 summarizes their contents.

Figure 2 displays the spatial distribution of our BCs in both Clouds. The bar of the LMC is clearly visible, although $\mathrm{FO} / \mathrm{F}$ and $\mathrm{SO} / \mathrm{FO}$ objects are widely distributed in both the LMC and SMC. We note a surdensity of Cepheids in the northern part of the $\operatorname{LMC}\left(75^{\circ}<\alpha<90^{\circ},-68^{\circ}<\delta<-66^{\circ}\right)$, corresponding to a region of star formation. We have checked that BCs have a spatial distribution similar to that of single-mode Cepheids by performing Kolmogorov-Smirnov (KS) tests using the stats package of $\mathrm{R}$, a language and environment for statistical computing ( $\mathrm{R}$ Development Core Team 2008). We computed the mean coordinates of the barycentre for the subsamples of $\mathrm{F}$ and FO objects, obtained to first approximation from a visual cut in the period-luminosity relation, and for the BCs. We then calculated the distance to the barycentre for each object and made the onesided KS tests reported in Table 2 on the resulting distance distributions. 

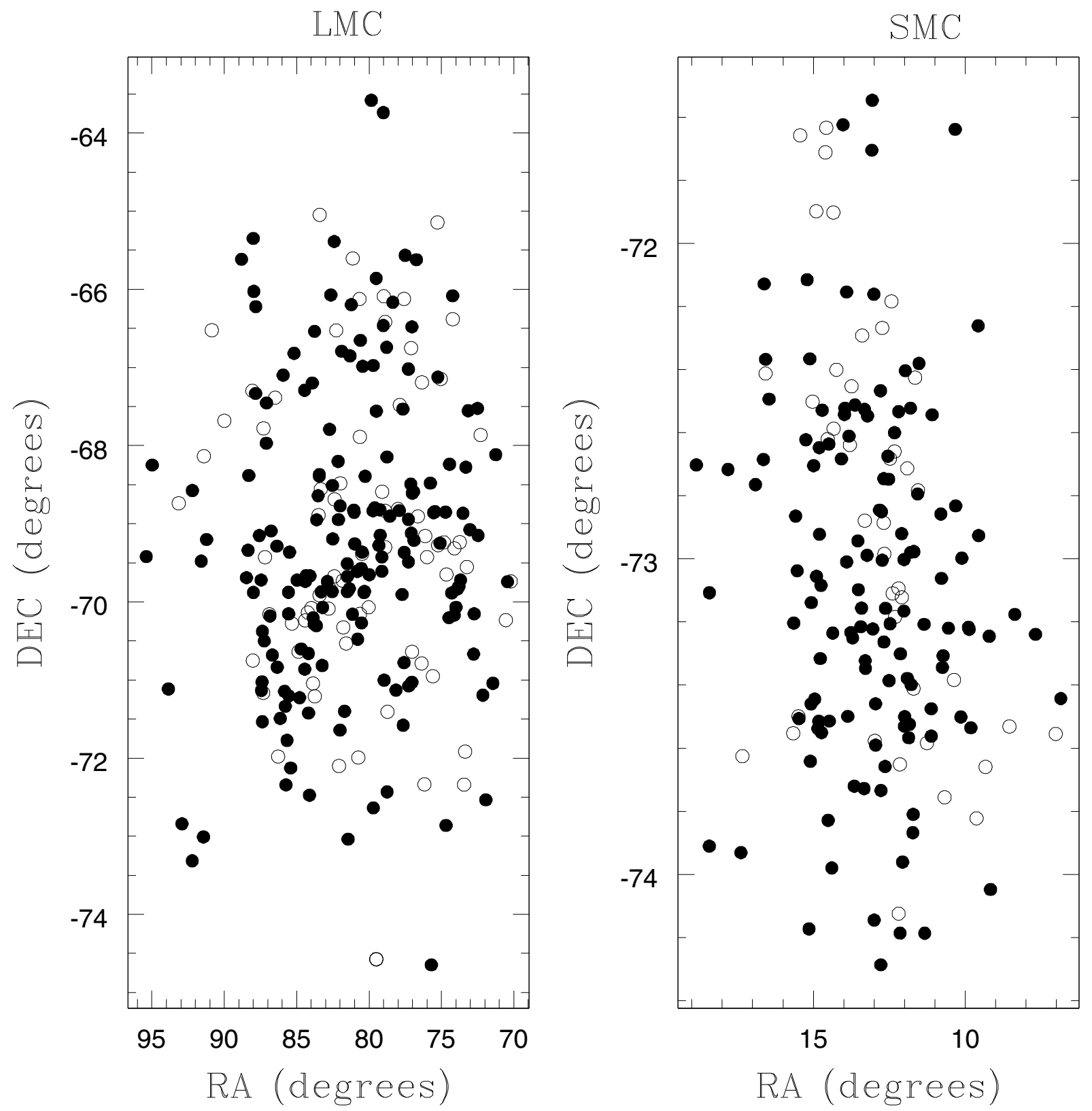

Fig. 2. Spatial distribution of BCs in the Magellanic Clouds. Open circles: FO/F objects; filled circles: SO/FO objects.

We first verified that the distributions of $\mathrm{F}$ and FO Cepheids are almost identical (first and third lines in Table 2). Note however the relatively high $p$-value (i.e. the probability to reject the null hypothesis) of this KS test in the LMC. The low $p$-values in the second and fourth lines in Table 2 show that BCs and single mode Cepheids are distributed similarly. The $D$ statistic represents the maximum distance between the distance distributions of populations (a) and (b).

We have compared our findings with the most recent studies from the OGLE group. Table 3 summarizes the numbers of stars which are in common between EROS-2 and OGLE, and those which were found only by EROS-2 or OGLE, respectively. For both the $\mathrm{FO} / \mathrm{F}$ or $\mathrm{SO} / \mathrm{FO}$ samples, the first column list the number of objects detected by both surveys, while the next two columns give the number of stars that are unique to each. OGLE data for the LMC are from Soszynski et al. (2008), for the SMC from Udalski et al. (1999). Although a detailed analysis of this comparison is beyond the scope of the present paper, it appears
Table 2. Results of the one-sided Kolmogorov-Smirnov tests performed on the spatial distributions of two different sets of Cepheids, noted (a) and (b). See text for further detail.

\begin{tabular}{ccccc}
\hline \hline & Set (a) & Set (b) & $D^{b}$ & $p$-value \\
\hline LMC & F (1772) & \\
& F + FO (2679) & FO/F + SO/FO (247) & 0.0994 & 0.1660 \\
\hline SMC & F (1256) & FO (1281) & 0.0245 \\
& F + FO (2537) & FO/F + SO/FO (161) & 0.1322 & 0.0335 \\
\hline
\end{tabular}

${ }^{a}$ Numbers in parentheses are the sizes of the populations; ${ }^{b} D$ defines the maximum distance between the distance distributions of (a) and (b); ${ }^{c}$ the $p$-values are the probability that the distributions are different.

that a large fraction of BCs are detected by both experiments, especially in the LMC for which an extended OGLE-III sample is available (Soszynski et al. 2008). The present work has, however, significantly increased the known samples of BCs, by some $31 \%$ in the LMC and $110 \%$ in the SMC. 
Table 3. Comparison of the EROS-2 (this work) and OGLE BC samples in the LMC and SMC.

\begin{tabular}{ccccccc}
\hline \hline & \multicolumn{3}{c}{ FO/F } & \multicolumn{3}{c}{ SO/FO } \\
\cline { 2 - 7 } & EROS-2 \& OGLE & EROS-2 & OGLE & EROS-2 \& OGLE & EROS-2 & OGLE \\
\hline LMC & 45 & 29 & 16 & 120 & 53 & 83 \\
SMC & 18 & 23 & 5 & 48 & 81 & 23 \\
\hline
\end{tabular}

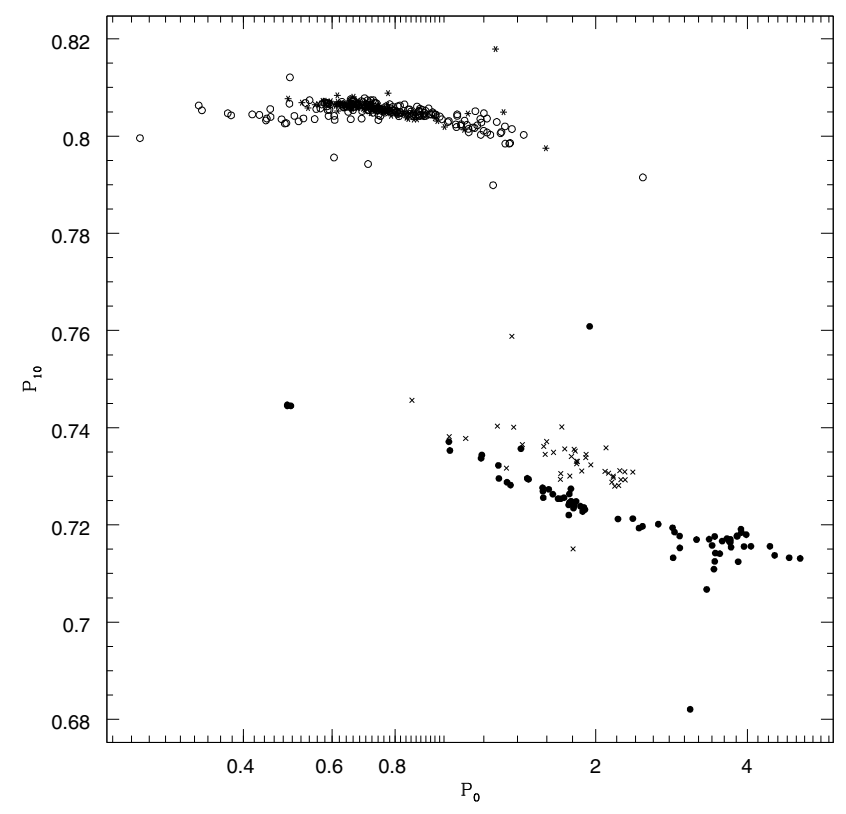

Fig. 3. Petersen diagram of FO/F EROS-2 BCs (lower; filled dots: LMC; crosses: SMC) and SO/FO objects (upper; open circles: LMC; stars: SMC).

There can be many reasons why some objects appear in the EROS-2 data and not in OGLE and vice versa. E.g., the spatial coverage is not the same, and the EROS-2 photometry is PSFbased while OGLE uses a differential image analysis method. In addition, the periodicity search algorithms are different and it should be useful to study whether they are sensitive to different numerical effects or not. In the LMC, $66(22 \mathrm{FO} / \mathrm{F}$ and $42 \mathrm{SO} / \mathrm{FO}$ ) of the 82 objects that are detected by EROS- 2 only are located in regions out of the OGLE-III fields. In the SMC, 69 (17 FO/F and $52 \mathrm{SO} / \mathrm{FO})$ of the 81 objects identified only by EROS-2 are external to the OGLE-II fields. Inversely, on the 127 BCs found by OGLE only, we estimated than $40 \%$ are located either outside or on the edge of the EROS-2 CCDs, which did not allow a proper photometric follow-up. For the $60 \%$ remaining, a mix between EROS-2 lower photometric accuracy and difference in algorithm sensitivity is thought to be the answer. Clearly, this question should be examined in more details in the future.

Figure 3 displays the period ratio $P_{10}=P_{1} / P_{0}$ vs. $\log P_{0}$, a Petersen diagram with all FO/F and SO/FO EROS-2 objects. While $\mathrm{SO} / \mathrm{FO}$ Magellanic objects are mixed in the upper part of the diagram, a clear separation between FO/F LMC and SMC $\mathrm{BCs}$ is visible in the lower part. This was previously noted by Beaulieu et al. (1997) who explained from a theoretical point of view that the observed period ratio results from a combination of two opposite effects: (i) the lower the metallicity, the smaller the opacity bump which affects the pulsation; (ii) at lower metallicity the structure of the star changes: at fixed mass, the luminosity increases and, therefore, the period ratio decreases. Moreover, the position of the blue loop of an evolutionary track of a Cepheid with respect to the instability strip is different in the LMC and in the SMC because of their different metallicity. As a consequence, lower pulsation periods are favoured in the SMC, while the highest periods are found in the LMC.

The metallicity of FO/F BCs has been recently reexamined by Buchler \& Szabó (2007). It has been known for some time that the location of a $\mathrm{BC}$ in the Petersen diagram correlates strongly with metallicity $Z$. We plotted all our LMC and SMC BCs on such a Petersen diagram in Figs. 4 and 5.

Buchler \& Szabó (2007) developed a convenient tool for determining metallicity limits, $Z_{\min }$ and $Z_{\max }$ from the location of a $\mathrm{BC}$ in the Petersen diagram, based only on accurate observed pulsation periods. From linear nonadiabatic convective stellar models they constructed a composite Petersen diagram (Figs. 4 and 5), in which the isometallicity lines delimit the lower and upper boundaries, respectively, of the range in which both $\mathrm{F}$ and FO modes are linearly unstable; this is the linear criterion for beat pulsations.

Thus, if a given BC falls below the upper period boundary for a certain value of $Z$ in Fig. 4, and above the corresponding lower period boundary for the same $Z$ in Fig. 5, then this is a possible value of $Z$ for this star. Thus, for example, for the LMC BC with $\log P_{0}=0.521$ and $P_{10}=0.7067$, Fig. 4 yields $Z_{\min } \sim$ 0.0077 , while Fig. 5 gives $Z_{\max } \sim 0.0093$. It is interesting to note that the observational data follow the theoretical isometallicity curves rather than a simple linear correlation.

In practice, one interpolates among the isometallicity lines with a simple code. Using this approach, we have computed the metallicities of our BCs, and give the mean $Z_{\text {mean }}$ together with $Z_{\min }$ and $Z_{\max }$ in the electronic tables. Figure 6 shows the distribution of mean metallicity for the $\mathrm{FO} / \mathrm{F} \mathrm{BCs}$ in the Magellanic Clouds. The size of the bins is $3.25 \times 10^{-4}$, corresponding to the mean uncertainties in $Z_{\text {mean }}$ which is $5.2 \times 10^{-4}$ in the LMC and $2.3 \times 10^{-4}$ in the SMC.

A comparison of these data with other determinations is not straightforward, because previous spectroscopic measurements such as those by Cole et al. (2005) or Cayrel de Strobel et al. (2001) report $[\mathrm{Fe} / \mathrm{H}]$ as obtained for stars from different populations, thus from different stages in the chemical evolution of the host galaxy than that of the young BCs. Also, we determine $Z$, not $[\mathrm{Fe} / \mathrm{H}]$, and allowance for the non-Solar abundance ratios in the LMC and SMC (Pompéia et al. 2008) would be required to make a direct comparison.

Three low-luminosity objects with periods $\sim 0.5 \mathrm{~d}$ fall at the left boundary of the Petersen diagrams: $\operatorname{lm} 0316 \mathrm{~m} 27403$, $\operatorname{lm} 0470 \mathrm{n} 7559$ and $\operatorname{lm} 0682 \mathrm{~m} 15849$ (Fig. 3). They also appear to have $Z>0.026$, which is not realistic. This therefore seems to rule out that they are very low mass Cepheids in the first crossing phase, as argued by Baraffe et al. (1998). In fact, they may not be bona fide Cepheids within the range of our calculated models. However, we recall that the only assumption in our computations 


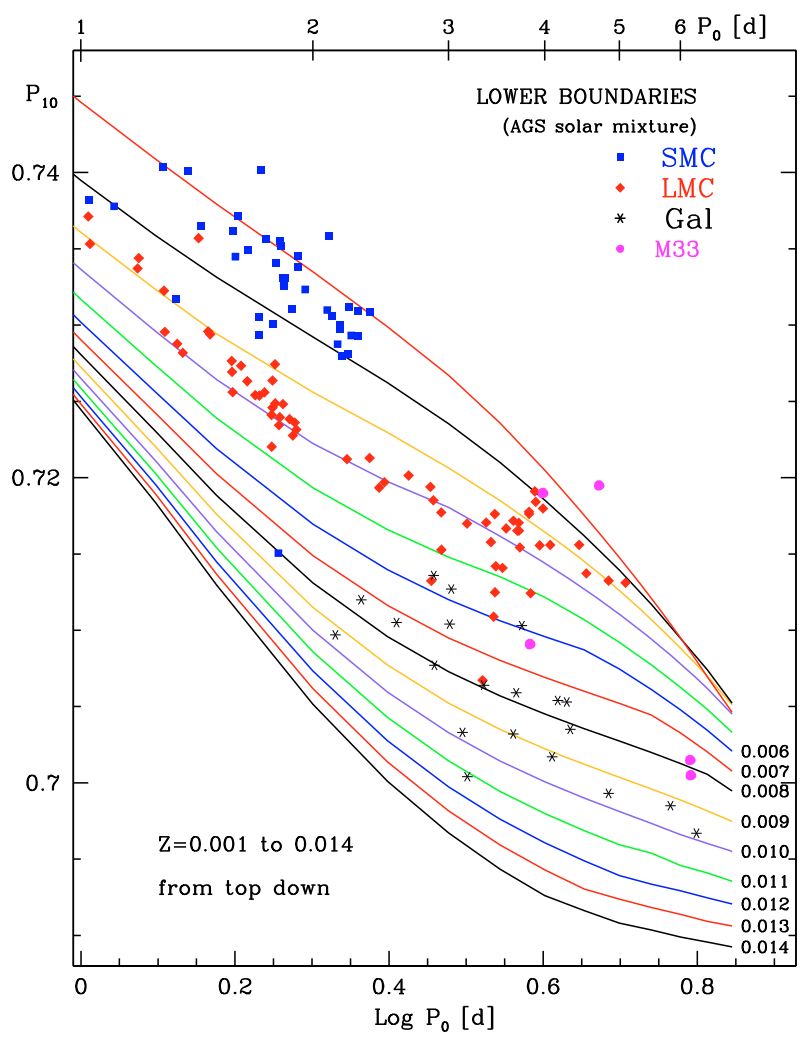

Fig. 4. Composite Petersen diagram $\left(P_{10}=P_{1} / P_{0}\right.$ vs. $\left.P_{0}\right)$ of the $\mathrm{FO} / \mathrm{F}$ BCs from EROS-2 compared to other known stars. The lines delimit the periods for which both $\mathrm{F}$ and FO are linearly unstable at given $Z$ (lower boundary in $Z$ for given $P_{10}$ and $P_{0}$ ). The metallicity increases downward from $Z=0.001$ (top line) to 0.014 in steps of 0.001 . Red rhombuses: EROS-2, LMC; blue squares: EROS-2, SMC; black stars: Galactic objects (Samus et al. 2004); pink circles: M33 objects (Beaulieu et al. 2006).

is that they are centrally condensed, like Cepheids, so that they can be represented by envelope models. It could therefore be that these stars are less evolved. Concerning the 4 BCs (2 LMC +2 SMC) not represented on Figs. 4 and 5 as they are outside the range of $P_{10}$ on that figures, we did not find any particular behaviour. Therefore we suggest, that these BCs may be used to localized zone in the Magellanic Clouds of lower and higher metallicity than average.

\section{Discussion of the uncertainties in $Z$}

We comment here on the three main sources of uncertainty in the inferred metallicities.

\subsection{The use of a linear Beat Cepheid criterion}

In Fig. 7 we show the derived $Z$ values as a function of $\log P_{0}$. The error bars reflect the consequences of using the linear criterion for beat behavior, i.e. simultaneous instability in the fundamental and first overtone modes. A comparison of Figs. 4 and 5, which show the upper and lower boundaries of $P_{10}$ as a function of $Z$, indicates that the uncertainties in $Z$ become very large for the longer-period BCs. It is indeed well known that the linear BC criterion is far too relaxed, so that real BCs occupy only a subregion of the corresponding parameter space (Stellingwerf 1974; Buchler \& Kovacs 1986).

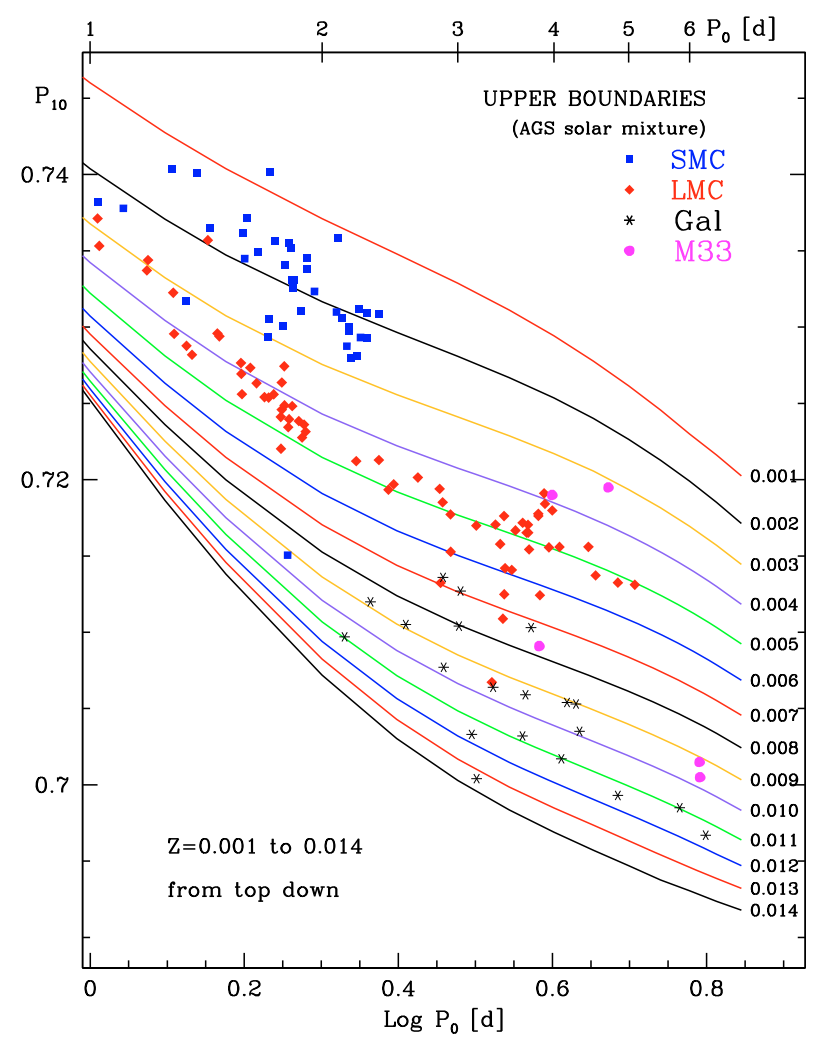

Fig. 5. As Fig. 4 (same symbols), but showing the upper boundary in $Z$ for which models are unstable for given $P_{0}$ and $P_{10}$.

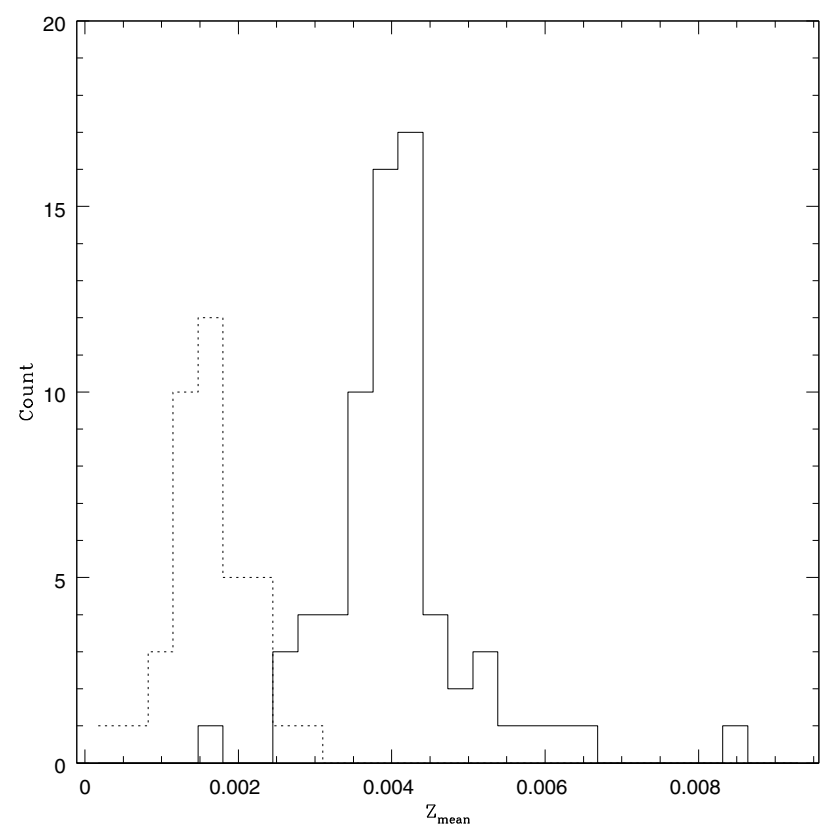

Fig. 6. Distribution of mean metallicities of FO/F BCs in the Magellanic Clouds. Solid line: LMC; dashed line: SMC. The bin size is $3.25 \times 10^{-4}$, corresponding to the mean error of $Z_{\text {mean }}$.

The obvious remedy is to perform nonlinear BC model calculations. This is a very computer intensive and time consuming endeavour, but is now under completion (Szabó \& Buchler 2009, in preparation). The work to date shows that the width of the double-mode instability strip is narrower by about a factor of 5 

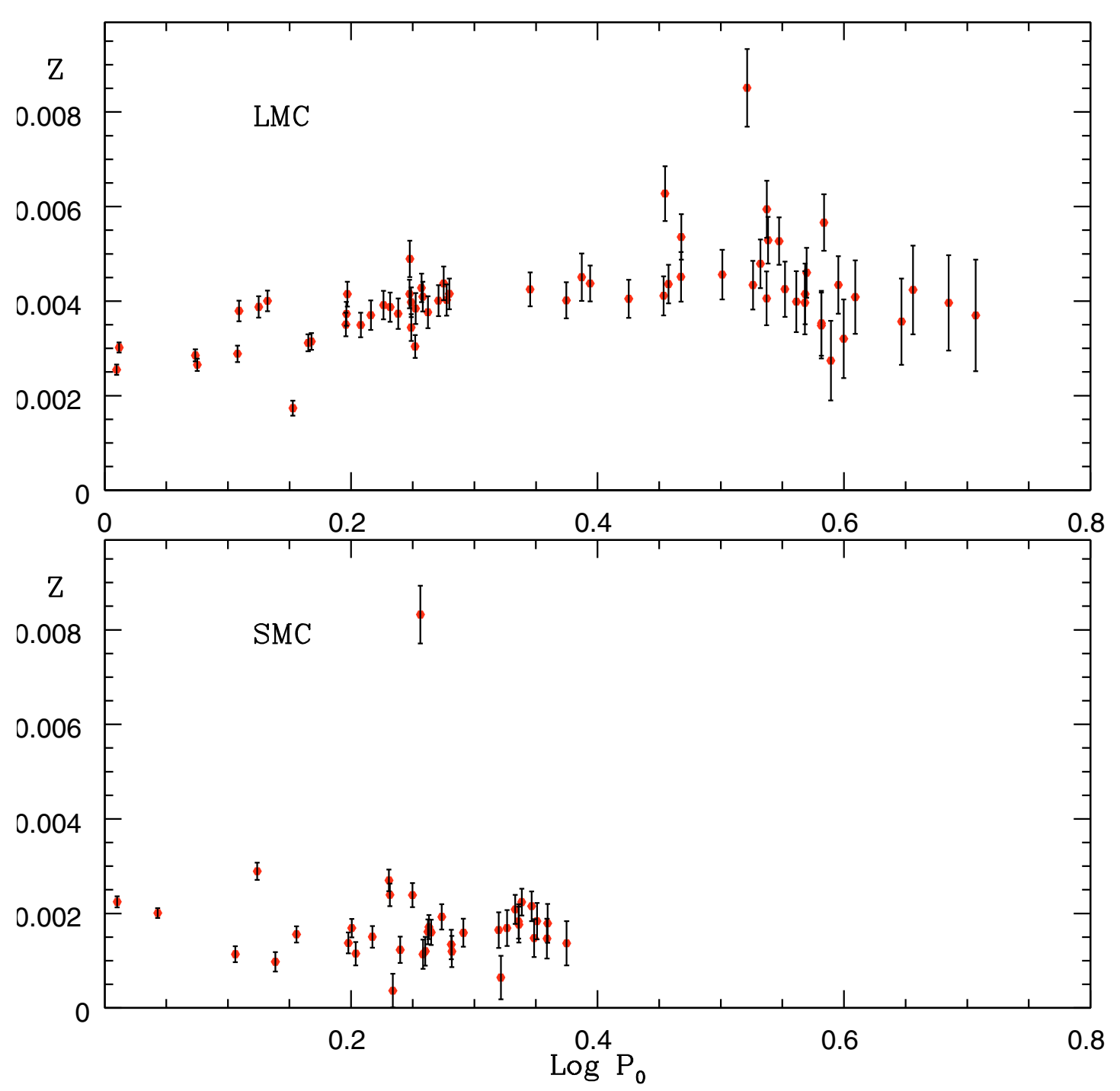

Fig. 7. Plot of the metallicity $Z$ vs. the period of the fundamental mode. Error bars are derived from the lower and upper boundaries in Figs. 4 and 5 .

in $\log T$ in a theoretical HR diagram. In a diagram like Fig. 5 this translates into a spread in $P_{10}$ of less than 0.0005 ; moreover, this is relatively uniform in $\log P_{0}$, i.e. does not open up towards the long periods as with the linear criterion. It is also found that, overall, the nonlinear range is closer to the upper linear boundary (Fig. 5) than the lower one (Fig. 4). On average, the nonlinear values of $Z$ are therefore expected to be somewhat higher than the linear ones.

\subsection{The chemical composition}

Our models use OPAL opacities computed with the revised solar composition of Asplund et al. (2005). This causes a substantial change in the inferred metallicity as compared to that obtained with the older Grevesse \& Noels (1993) solar composition used by Buchler (2008). The inferred average ("linear") $Z$ in the LMC is reduced from 0.0070 to 0.0044 and that of the SMC from 0.0028 to 0.0018 . This is of course a gross overestimate of the true uncertainty in metallicity, because any future revision of the composition is expected to be smaller than the difference between Asplund et al. (2005) and Grevesse \& Noels (1993). We also note that Magellanic Cloud stars in general do not have Solar abundance ratios (Pompéia et al. 2008) as we have assumed in our models, and we do not know how much this might change the results.

\subsection{Modelling uncertainties}

Buchler (2008) has shown that the approximation of envelope models breaks down for the short-period Cepheids, and that it is necessary to compute full stellar models (which we are not equipped to do at this stage). One may therefore wonder whether the lower metallicity at shorter periods seen in Fig. 7 is a result of this uncertainty in the models, or whether there is a physical or evolutionary reason for the lower metallicity, e.g. Cordier et al. (2003).

\section{Conclusion}

We have reported a new survey of the BC populations in the Magellanic Clouds which complements the recent work of the OGLE group in the SMC (Soszynski et al. 2008) and extends it over a larger area in the LMC. Our new, large FO/F sample yields a new metallicity distribution for another stellar population in the LMC and the SMC. Together with previous studies of HII regions, planetary nebulae or red giants, this contributes to a 
more complete view of the metallicity of the Magellanic Clouds and its links to stellar evolution. We deduce from the present data a mean metallicty of 0.0045 in the LMC and 0.0018 in the SMC.

The demonstration that such BC stars are a useful and independent way to determine metallicities has considerable promise for future studies in the Local Group. In M33, 5 BCs were found among a sample of more than 2000 Cepheids (Beaulieu et al. 2006), and we have shown here that tens of BCs can be detected in the Magellanic Clouds, among a total population of 2000-3000 single-mode Cepheids (see Soszynski et al. 2008 and Table 2 above). The frequency of BCs in other galaxies of the Local Group is likely to be between these values. M31 is the obvious first candidate for such a search.

Acknowledgements. J.P.B., J.B.M. and A.S.C. acknowledge financial support from the European Associated Laboratory "Astrophysics Poland-France". J.R.B. and A.S.C. gratefully acknowledge, respectively, the support of NSF and KBN (grants AST 07-07972 and OISE 04-17772 at UF, and 1P03D 02529 at UAM). R.Sz. acknowledges the support of a Hungarian Eötvös Fellowship. R.Sz. and J.R.B. are grateful to the Hungarian NIIF Supercomputing Facility and to the UF High-Performance Computing Center for providing computational resources and support.

\section{References}

Alcock, C., Allsman, R. A., Axelrod, T. S., et al. 1995, AJ, 109, 1653

Alcock, C., Allsman, R. A., Alves, D., et al. The MACHO Collaboration 1999, ApJ, 511, 185

Ansari, R. 1996, Vist. Astron., 40, 519

Asplund, M., Grevesse, N., \& Sauval, A. J. 2005, in Cosmic Abundances as Records of Stellar Evolution and Nucleosynthesis, ed. T. G. Barnes, III, \& F. N. Bash, ASP Conf Ser., 336, 25

Baraffe, I., Alibert, Y., Méra, D., Chabrier, G., \& Beaulieu, J.-P. 1998, ApJ, 499, L205

Beaulieu, J.-P., Buchler, J. R., Marquette, J.-B., Hartman, J. D., \& Schwarzenberg-Czerny, A. 2006, ApJ, 653, L101

Beaulieu, J. P., Grison, P., Tobin, W., et al. 1995, A\&A, 303, 137

Beaulieu, J. P., Krockenberger, M., Sasselov, D. D., et al. 1997, A\&A, 321, L5

Buchler, J. R. 2008, ApJ, 680, 1412

Buchler, J. R., \& Kovacs, G. 1986, ApJ, 308, 661

Buchler, J. R., \& Szabó, R. 2007, ApJ, 660, 723

Carrera, R., Gallart, C., Aparicio, A., et al. 2008a, AJ, 136, 1039

Carrera, R., Gallart, C., Hardy, E., Aparicio, A., \& Zinn, R. 2008b, AJ, 135, 836

Carrera, R., Gallart, C., Pancino, E., \& Zinn, R. 2007, AJ, 134, 1298

Cayrel de Strobel, G., Soubiran, C., \& Ralite, N. 2001, A\&A, 373, 159

Cole, A. A., Tolstoy, E., Gallagher, III, J. S., \& Smecker-Hane, T. A. 2005, AJ, 129,1465

Cordier, D., Goupil, M. J., \& Lebreton, Y. 2003, A\&A, 409, 491

Derue, F., Marquette, J.-B., Lupone, S., et al. 2002, A\&A, 389, 149

Grevesse, N., \& Noels, A. 1993, in Origin and Evolution of the Elements, ed. N. Prantzos, E. Vangioni-Flam, \& M. Casse, 14

Idiart, T. P., Maciel, W. J., \& Costa, R. D. D. 2007, A\&A, 472, 101

Kovács, G. 2008, ArXiv e-prints, 08024166

Leisy, P., \& Dennefeld, M. 2006, A\&A, 456, 451
Petersen, J. O. 1973, A\&A, 27, 89

Pompéia, L., Hill, V., Spite, M., et al. 2008, A\&A, 480, 379

R Development Core Team 2008, R: A Language and Environment for Statistical Computing, R Foundation for Statistical Computing, Vienna, Austria

Samus, N. N., Durlevich, O. V., et al. 2004, VizieR Online Data Catalog, 2250, 0

Schwarzenberg-Czerny, A. 2003, in Interplay of Periodic, Cyclic and Stochastic Variability in Selected Areas of the H-R Diagram, ed. C. Sterken, ASP Conf. Ser., 292, 383

Simon, N. R., \& Lee, A. S. 1981, ApJ, 248, 291

Soszynski, I., Udalski, A., Szymanski, M., et al. 2000, Acta Astron., 50, 451

Soszynski, I., Poleski, R., Udalski, A., et al. 2008, ArXiv e-prints, 808

Stellingwerf, R. F. 1974, ApJ, 192, 139

Tisserand, P., Le Guillou, L., Afonso, C., et al. The EROS-2 Collaboration. 2007, A\&A, 469, 387

Udalski, A., Soszynski, I., Szymanski, M., et al. 1999, Acta Astron., 49, 1

1 Institut d'Astrophysique de Paris, UMR7095 CNRS, Université Pierre \& Marie Curie, 98 bis Boulevard Arago, 75014 Paris, France e-mail: marquett@iap.fr

2 Physics Department, University of Florida, Gainesville, FL 32611-8440, USA

3 Konkoly Observatory, Budapest, PO Box 67, 1525, Hungary

4 Research School of Astronomy \& Astrophysics, Mount Stromlo Observatory, Cotter Road, Weston ACT 2611, Australia

5 CEA, DSM, DAPNIA, Centre d'Études de Saclay,

91191 Gif-sur-Yvette Cedex, France

6 Observatoire Midi-Pyrénées, Laboratoire d'Astrophysique (UMR

5572), 14 av. E. Belin, 31400 Toulouse, France

7 Centrum Astronomiczne im. M. Kopernika, Bartycka 18,

00-716 Warszawa, Poland

8 Obserwatorium Astronomiczne, Uniwersytet A. Mickiewicza, Sloneczna 36, 60-286 Poznan, Poland

9 Max-Planck-Institut für Astronomie, Königstuhl 17,

69117 Heidelberg, Germany

10 Laboratoire de l'Accélérateur Linéaire, IN2P3 CNRS, Université de Paris-Sud, 91405 Orsay Cedex, France

11 The Niels Bohr Institute, Copenhagen University, Juliane Maries Vej 30, DK2100 Copenhagen, Denmark

12 Department of Astronomy, Ohio State University, Columbus, $\mathrm{OH}$ 43210, USA

13 Division of Medical Imaging Physics, Johns Hopkins University, Baltimore, MD 21287-0859, USA

14 LPNHE, CNRS-IN2P3 and Universités Paris 6 \& Paris 7, 4 place Jussieu, 75252 Paris Cedex 05, France

15 Observatoire Astronomique de Strasbourg, UMR 7550, 11 rue de l'Université, 67000 Strasbourg, France

16 Observatoire de Marseille, 2 Place Le Verrier, 13248 Marseille Cedex 04, France

17 European Southern Observatory (ESO), Casilla 19001, Santiago 19, Chile

18 San Pedro de Atacama Celestial Exploration, Casilla 21, San Pedro de Atacama, Chile 